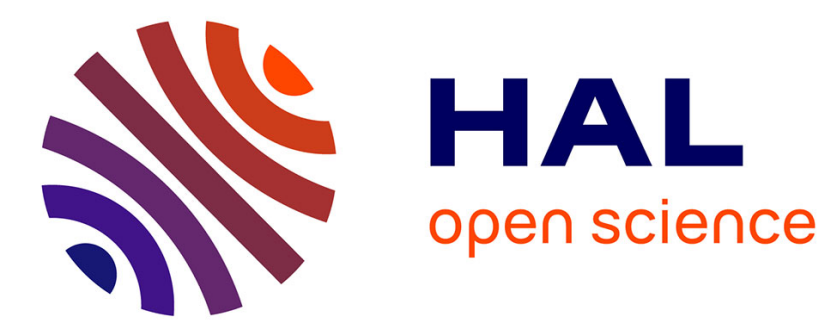

\title{
Training-based on real-time motion evaluation for functional rehabilitation in virtual environment
}

Van-Hanh Nguyen, Frédéric Merienne, Jean-Luc Martinez

\section{To cite this version:}

Van-Hanh Nguyen, Frédéric Merienne, Jean-Luc Martinez. Training-based on real-time motion evaluation for functional rehabilitation in virtual environment. International Journal on Image and Graphics, 2010, 10 (2), pp.235-250. 10.1142/S0219467810003755 . hal-01110998

\section{HAL Id: hal-01110998 \\ https://hal.science/hal-01110998}

Submitted on 29 Jan 2015

HAL is a multi-disciplinary open access archive for the deposit and dissemination of scientific research documents, whether they are published or not. The documents may come from teaching and research institutions in France or abroad, or from public or private research centers.
L'archive ouverte pluridisciplinaire HAL, est destinée au dépôt et à la diffusion de documents scientifiques de niveau recherche, publiés ou non, émanant des établissements d'enseignement et de recherche français ou étrangers, des laboratoires publics ou privés. 


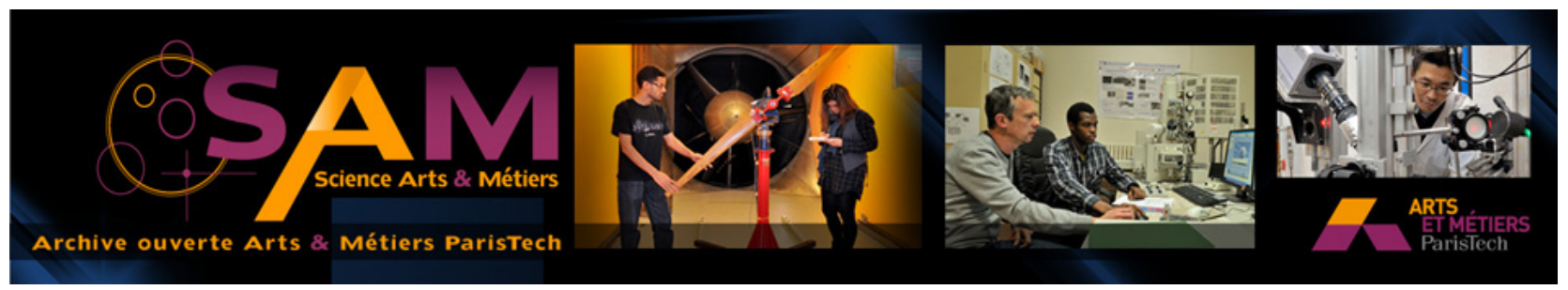

Science Arts \& Métiers (SAM)

is an open access repository that collects the work of Arts et Métiers ParisTech researchers and makes it freely available over the web where possible.

This is an author-deposited version published in: http://sam.ensam.eu

Handle ID: .http://hdl.handle.net/10985/9283

\section{To cite this version :}

Van-Hanh NGUYEN, Frédéric MERIENNE, Jean-Luc MARTINEZ - Training-based on real-time motion evaluation for functional rehabilitation in virtual environment - International Journal on Image and Graphics - Vol. 10, n², p.235-250 - 2010 


\title{
TRAINING BASED ON REAL-TIME MOTION EVALUATION FOR FUNCTIONAL REHABILITATION IN VIRTUAL ENVIRONMENT
}

\author{
VAN-HANH NGUYEN*, FREDERIC MERIENNE ${ }^{\dagger}$ \\ and JEAN-LUC MARTINEZ \\ Arts et Metiers Paris Tech, CNRS \\ LE2I - Institut Image, 2 rue Thomas Dumorey \\ 71100 Chalon-sur-Saone, France \\ *hanhnv@gmail.com \\ †frederic.merienne@ensam.eu \\ ${ }^{\ddagger}$ jean-luc.martinez@cluny.ensam.fr
}

\begin{abstract}
One of the most effective applications of virtual reality (VR) in physical rehabilitation is training, where patients are trained for sequence decision-making in special situations presented in virtual environment. In this application, the evaluation of the movement of the subject performing a physical task is crucial. A good evaluation of the motion is necessary to follow the progression of the patient during his training session. Therefore, it helps therapist to better supervise therapeutic planning. Actually, the performance of the patient's training is determined by subjective observation of the therapist. Our approach is to propose a system that allows the patient to perform his training and to evaluate the progress of training in an autonomous way. This system consists of a motion analysis technique for a rehabilitation application where the patient is represented by his own avatar in virtual environment. The task performance required from the patient is his capability to reproduce in real time a movement. The real-time motion evaluation technique is based on the time series data matching method called Longest Common Sub-Sequence (hereafter LCSS). It is used to calculate distance between the reference motion of virtual avatar and the captured motion data of the patients and thus is used to determine how well the patients are doing during the training. The complexity of the technique proposed is in the order of $\mathrm{O}(\delta)$ in which $\delta$ is a constant matching window size. Our prototype application is based on Tai-chi movements which have shown many health benefits and are increasingly used for therapeutic purposes.
\end{abstract}

Keywords: Virtual training; real-time LCSS; motion evaluation; motor rehabilitation.

\section{Introduction}

Many current and potential applications for human activities are developed through VR systems involving virtual human or virtual avatar. ${ }^{18,19}$ Several popular 
applications are involved in computer game, simulation based training and learning, virtual patient for surgery, plastic surgery and virtual psychotherapy. The use of virtual avatar increases the natural interaction within virtual environment. The users' more natural perception of each other (and of autonomous actors) increases their sense of being together, and thus the overall sense of shared presence in the environment. ${ }^{1}$ In the application of virtual rehabilitation, there have been an increasing number of papers dedicated to the approach of using a virtual avatar. Actually, the purpose of using a virtual avatar in rehabilitation is to guide a patient in performing therapeutic training. Therapeutic training consists of asking the patient to redo a sequence of actions with various scenarii presented by the VR training system. ${ }^{2}$ The sequence of actions is usually designed to learn performing the tasks. In this approach, a critical issue is the evaluation of the effective motion of the subject in realizing his task. Effectively, an ideal movement of the patient in physical rehabilitation tends to approach the movement of the healthy person. Moreover, movement in a virtual environment is equivalent to a muscular execution in the physical environment. ${ }^{20,21}$ Therefore, the knowledge of a patient performing the task compared to a healthy person could deduce the progress of therapeutic planning. Hence, it may help therapist to better supervise the planning of therapy.

In this paper, we investigate the use real-time system for evaluating the movement carried out by patients during the rehabilitation training task. In our approach, the task consists of replicating a movement presented by a virtual avatar in real-time. The task performance is measured by the similarity between the motion replicated by the patient and the guide motion executed by a virtual avatar. The similarity of the two motions can be measured by different ways: similarity based position/orientation and speed-based similarity. Some other works measure the performances separately and calculate the overall rating after that. ${ }^{4,2}$ In our system, both of these aspects of performance are measured in one time. This allows obtaining similarity value based on key frame by key frame comparison between the reference and the captured motion data. This comparison is implemented based on the algorithm called Longest Common Sub-Sequence (LCSS) which is an effective algorithm for time series matching. In fact, real-time similarity is the number of similar key frames between patient's motion and virtual avatar's motion.

In the next section of the paper, we review related research work focused on motion evaluation and the algorithms for time series matching. In addition, we present the advantages of LCSS algorithm in the case of human motion matching compared to other algorithms. Section 3 gives an overall configuration of the proposed training system based real-time motion evaluation. In Sec. 4, we describe the LCSS algorithm for real-time motion evaluation. Section 5 presents the prototype system, our experiment consisting of a Tai-Chi learning session and the obtained results and discussion. In Sec. 6 the conclusion and research perspectives are presented. 


\section{Related Work}

The measurement of body movement for rehabilitation purpose requires the definition of the representation of human body structure. There are some standards for human skeleton structure among which H-ANIM $1.1^{10}$ is the most popular and used for Cal3D library used in our work for avatar rendering. H-ANIM 1.1 defines the human body as a number of segments (such as the forearm, hand and foot) which are connected to each other by joints (such as the elbow, wrist and ankle). Each joint in the body is represented by a joint node used to define the relationship of each body segment to its immediate parent. To define the representation of each joint of animated human body, Cardle $^{5}$ reviewed some human representations and compared their characteristics such as rotation invariant, interpolation quality, computational cost and weights required. Finally, he proposed to use the angle-axis representation for the joints.

A joint is represented in axis-angle as: $P(x, y, z, w)$ or $P\left(w_{1}, w_{2}, w_{3}, 1\right)$ in a more compact representation, where $w_{1}=x \cdot w, w_{2}=y \cdot w, w_{3}=z \cdot w$. We can define the distance between two joints represented in axis-angles as follows ${ }^{7}$ :

$$
d\left(P_{1}, P_{2}\right)=\sum_{i=1}^{3}\left(w_{i}^{1}-w_{i}^{2}\right)^{2}
$$

Having defined the joints structure and joint representation, we can define the motion or animation in the case of the study reported in this paper. An animation is a sequence of key-frames, a key-frame is defined as a set of position and orientation values of human skeleton joints. A general formula of animation $Q$ that includes $m$ key-frames of $D$-joints can be described as follows:

$$
Q=\left\{\left(q_{0}^{1}, q_{0}^{2}, \ldots, q_{0}^{D}\right\},\left(q_{1}^{1}, q_{1}^{2}, \ldots, q_{1}^{D}\right), \ldots,\left(q_{m}^{1}, q_{m}^{2}, \ldots, q_{m}^{D}\right\}\right\}
$$

Actually, most of the studies proposed in human motion evaluation are made in offline mode. Thus, the measurement of the similarity between two human motions is performed on the basic of pre-recorded data of full motions. In our work, we develop a real time similarity method to compare the motion carried out by a patient with a correct motion executed by an expert. In order to carry out this comparison we utilize the technique of data retrieval which has many applications in the domain of time series data mining. We can consider the work of Takeshi Yabe and Katsumi Tanaka ${ }^{4}$ who used the Dynamic Programming matching method to measure the similarity between the user motion and a teacher's model motion for whole body as multi-stream time series data. In others hand, using motion evaluation to analyze the similarities and varieties in human motion trajectories of predefined grasping and disposing movement is another example. ${ }^{3}$ In terms of VR-based training purpose, the study of Baek, Lee and $\mathrm{Kim}^{2}$ measured the similarity between motions to provide the trainee with advice to improve training. The offline-mode study of Cardle ${ }^{5}$ is very close to our approach. He used the same joint structure, the representation and the algorithm to measure similarity. However, the study of 


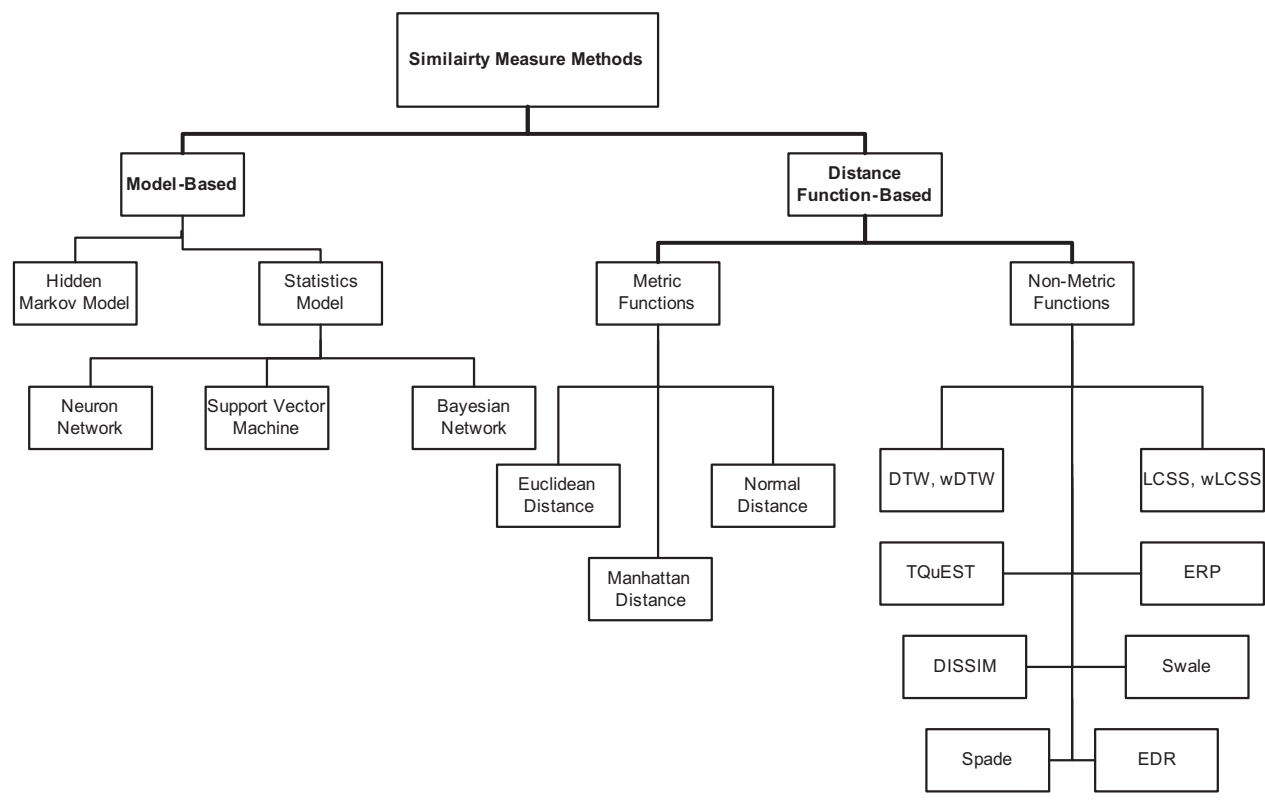

Fig. 1. Category of similarity measurement methods classified in two approaches: Model-Based and Distance Functions-Based.

Cardle focused on motion editing rather than motion evaluation. Motion evaluation is part of time series data matching problem which is studied in data mining with very large database (VLDB). To solve this problem, there are many proposed methods classified in two main approaches illustrated in Fig. 1.

With the multitude of proposed techniques, there is a strong need to determine the most effective method to be used for time series data matching problem. Ding et al. (2008) performed a comparison of the major techniques of distance functionbased approach by testing their effectiveness on 38 time series data set from a wide variety of application domains. ${ }^{6}$ Experimental results obtained show that, in over a dozen of distance measures of similarity among time series data in the literature, wLCSS (weighted Longest Common Sub-Sequence) ${ }^{7-9}$ and wDTW ${ }^{10,11}$ (weighted Dynamic Time Wrapping) are slightly better than the others from the accuracy point of view. Moreover, on large data set, the computation cost of wLCSS and $\mathrm{wDTW}$ is very close to the Euclidean distance algorithm. The experimental result of Keogh ${ }^{12}$ had the objective to compare performance between two approaches, the model-based algorithm and the distance function based algorithm. This work showed that the performance of distance function based algorithm is much better than the performance of model-based algorithm, for the criteria of accuracy and computational cost. In taking into account these results, wLCSS and wDTW are used in the research reported in this paper.

Motion capture (MoCap) data, which is captured by recording the positions of the markers during the motion, is a type of time-series data. Unlike other types 
of time-series data (stock, weather, etc.), MoCap data has usually many outliers and noises mainly due to sensor failures, disturbance signals or errors in detection technique. By its characteristics, MoCap data consist of different sampling rates of tracking and recording devices combined with different speeds of moving objects. Therefore, we would like to use a distance function that can address the following issues $^{7}$ :

- Different sampling rates or different speed

- Different data length

- Capability of ignoring the outliers

- Robustness to noise

The wDTW technique processes all items of the MoCap data sequence. Consequently, it does not ignore outliers. Furthermore, this technique is sensitive to noise. However, wLCSS was applied to address these problems. ${ }^{17}$ Some published research works showed that wLCSS is more robust and appropriate to compare distance measures for MoCap data than wDTW. ${ }^{15}$ Figure 2 shows a comparison between wDTW and wLCSS techniquew. Indeed, wLCSS uses distance function in our work. Since the approach described in the paper is focused on real-time motion evaluation, the method used is called real-time wLCSS and noted rwLCSS.

\section{Training-Based Motion Evaluation System}

The overall configuration of our training-based real-time motion evaluation system is illustrated in Fig. 3. The system is composed by an infra-red motion capture system (A.R.Tracking) and a Cave Automatic Virtual Environment (CAVE). Full body motion of patients is captured by using a set of markers and animated simultaneously with the reference motion which is carried out from a motion database.

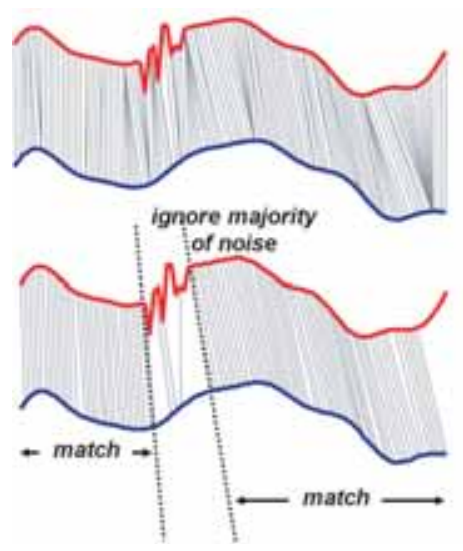

Disadvantages of DTW:

A. All points are matched

B. Outliers can distort distance

C. One-to-many mapping

Advantages of LCSS:

A. Outlying values not matched

B. Distance/Similarity distorted less

C. Constraints in time \& space

Fig. 2. Comparison between wDTW and wLCSS techniques. ${ }^{17}$ 


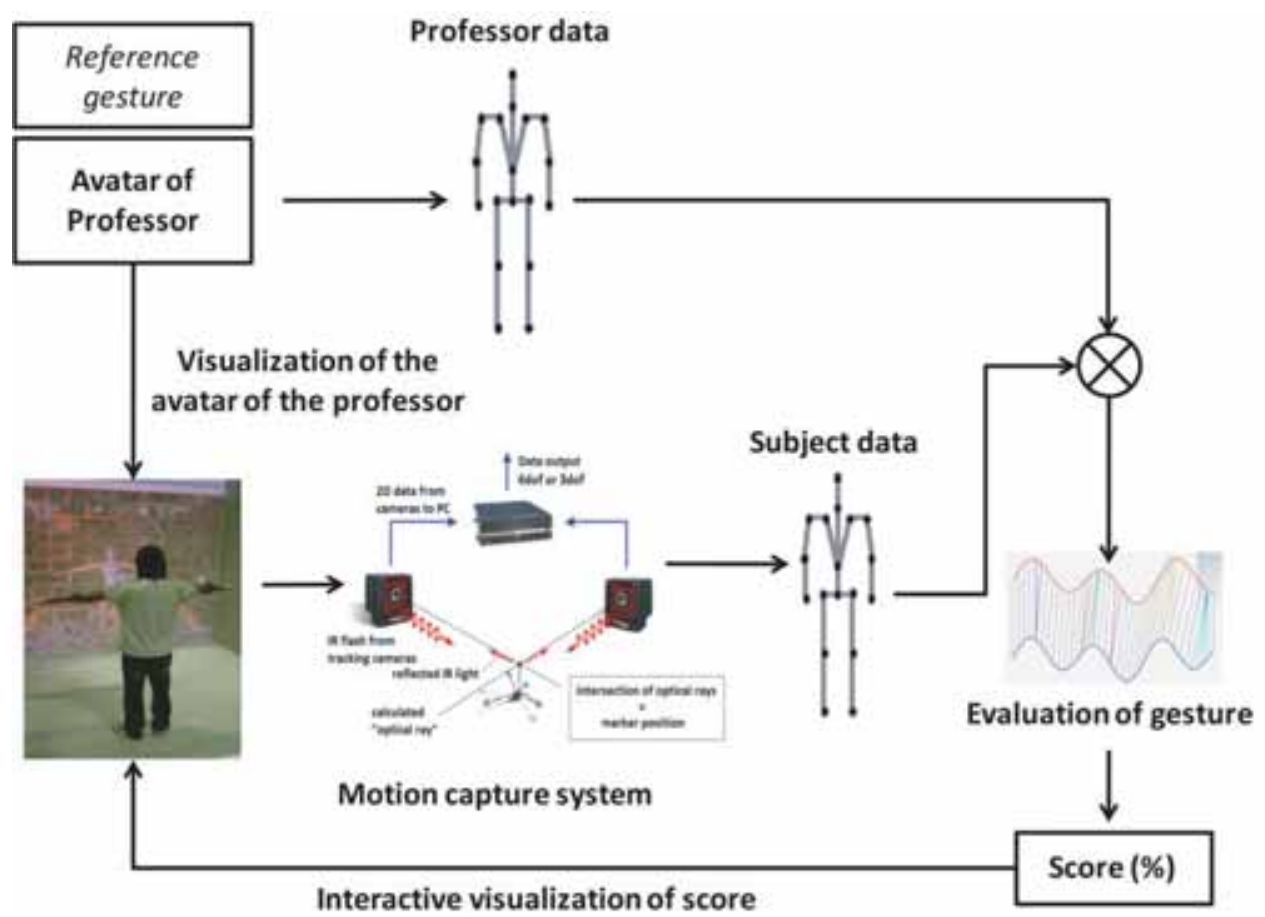

Fig. 3. Overall configuration of training-based real-time motion evaluation system.

Before training, a calibration procedure is carried out for each patient in order to locate the joints angle or Centers of Rotation ( CoRs) and 3D position of root joint (3DoR) from the 3D position of markers and to estimate the size of the virtual avatar which is based on the marker attached to the head. This technique is implemented using the work carried out by Cameron et al. ${ }^{13}$ who proposed a closed form sequential solution enabling real-time estimation of the CoRs in quaternion coordinates. This technique takes full advantages of the approximation that all markers on body segments are attached to a rigid body. ${ }^{14}$ To be used in motion evaluation, a simple transformation from quaternion CoRs to axis-angle is performed. The virtual avatar in our system is inherited from an available and easy-to-use library called Cal3D.

In this research work, the motion database is a Tai-Chi motions volume which is recorded by A.R. Tracking system and is interpolated to reach the frequency of $60 \mathrm{~Hz}$. The chosen scenario is as follows: the patient is watching the Tai-Chi motion performed by a Tai-Chi professor from a specific viewpoint which is rendered by a virtual avatar. The patient has to reproduce the Tai-Chi motion simultaneously. The system measures in real-time the distance between the two motions in the joint angles of key-frames which are represented in axis-angles. Due to different influences of joints on the human animation, we manually define the weights for each joint for the experiment. Parent joint are more weighted than child joint in general. 
Because the frequency of training progress can be different from $60 \mathrm{~Hz}$, we need to synchronize correctly the two motions. We compute the time needed to render the virtual scene and take this time into account to indicate the correct key-frames of the reference motion to render in the virtual scene. In real-time training, the motion of the trainee always starts later than the motion of avatar. The progress of the evaluation is completed when the temporal length of trainee's motion is equal to the duration of the motion of the virtual avatar. The similarity between the two motions is rendered by an indication of the percentage of similarity and a background color changing (green color for a good replication, yellow color for average and red color for bad replication).

\section{The Simple but Robust Algorithm for Real-Time Motion Evaluation: rwLCSS}

The rwLCSS algorithm is based on LCSS model that supports time shifting and unequal length sequence matching while ignoring noisy parts. Practically, by using LCSS to calculate the similarity between key-frames, motions that are close in space at different time instants can be matched when the time instants are also close. By using standard LCSS, the final similarity in online mode of motions evaluation is less than in offline mode. In online mode, the key-frame of the trainee's motion at a time instant is only compared to key-frames of the reference motion at prior times. Accordingly, with an aim to validate real-time LCSS, we developed a modified LCSS technique for offline mode in a way as equivalent to online mode. From standard recursive definition of $\operatorname{LCSS}^{9}$ over two multi-dimensional time-series motions $Q$ (query or reference motion) and $\mathrm{C}$ (candidate or replicated motion) with temporal length of $n$ and $m$, respectively, the modified LCSS is defined as:
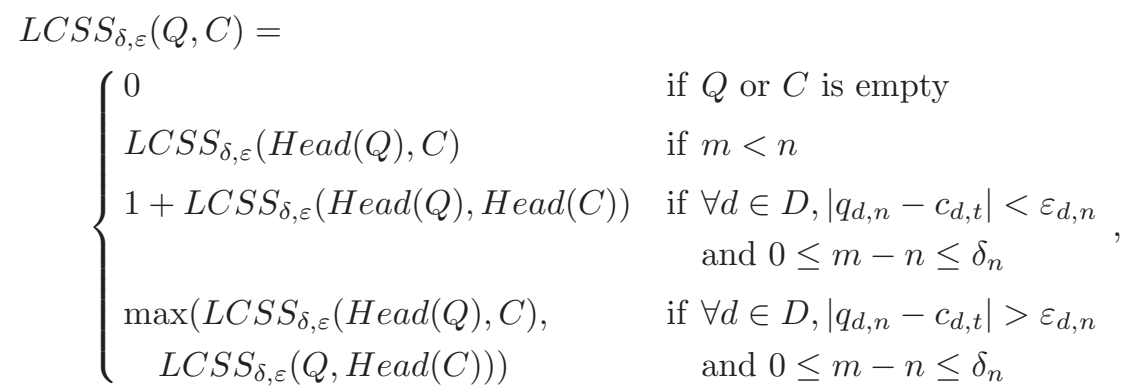

where $\delta \in \Re^{n}$ is temporal threshold, $\varepsilon \in \Re^{n x D}$ is the spatial threshold and $D$ is the total number of dimensions to match over. The complexity of LCSS model is in the order of $O(\delta(n+m))$ with a constant matching window $\delta$ in time. By using this modified LCSS, the final result of online mode is equivalent to the one of offline mode.

In our study of motion matching, each key-frame is a multi-dimensional timeseries in which D is the number of joints to match over. Due to the weight or the priority of joints discussed above, instead of using LCSS, we use wLCSS. Practically, 
wLCSS is the LCSS case when we define a different spatial threshold $\varepsilon$ for each joint. The more weighted joints will be defined with less value of $\varepsilon$ and vice-versa. In fact, the assignment of weights for each joint is equivalent to assign the spatial threshold in LCSS model. The final similarity between two time-series motions is defined as:

$$
D_{\delta, \varepsilon}(Q, C)=1-S_{\delta, \varepsilon}(Q, C) \quad \text { where } S_{\delta, \varepsilon}(Q, C)=\frac{L C S S_{\delta, \varepsilon}(Q, C)}{\min (n ; m)} .
$$

Based on modified $w L C S S$, we propose a real-time rwLCSS model.

$Q_{t}$ is the subsequence of the reference motion from the start to time instant $t$; $c_{t}$ is the key-frame of trainee's motion at time instant $t$;

$\operatorname{rwLCSS}\left(Q_{t}, c_{t}\right)$ is a function of the longest common subsequence between $Q_{t}$ and $\mathrm{c}_{t}$. The $\operatorname{rwLCSS}\left(Q_{t}, c_{t}\right)$ is defined recursively as follow:

$\operatorname{wrLCSS}\left(Q_{t}, c_{t}\right)= \begin{cases}0 & \text { if } Q_{t} \text { or } c_{t} \text { is empty } \\ 1+\operatorname{wr} \operatorname{LCSS}\left(\operatorname{Head}\left(Q_{t}\right), c_{t-1}\right) & \text { if } \forall d \in D,\left|q_{d, n}-c_{d, t}\right|<\varepsilon_{d} \\ \max \left(\operatorname{wr} \operatorname{LCSS}\left(\operatorname{Head}\left(Q_{t}\right), c_{t}\right),\right. & \text { otherwise } \\ \left.\operatorname{wr} \operatorname{LCSS}\left(\operatorname{Head}\left(Q_{t}\right), c_{t-1}\right)\right) & \end{cases}$

where, $n$ is the temporal length of $Q$ at time instant $t$.

The temporal and spatial complexity of rwLCSS model are in the order of $O(\delta)$ with a constant matching window $\delta$ and $O(n)$ with the dimension of reference motion $n$, respectively. Accordingly, the real-time similarity between $Q_{t}$ and $c_{t}$ is defined as follows:

$$
D_{\delta, \varepsilon}\left(Q_{t}, c_{t}\right)=1-S_{\delta, \varepsilon}\left(Q_{t}, c_{t}\right) \quad \text { where } S_{\delta, \varepsilon}\left(Q_{t}, c_{t}\right)=\frac{L C S S_{\delta, \varepsilon}\left(Q_{t}, c_{t}\right)}{n}
$$

\section{Prototype System and Experiment}

Our prototype system is based on motion capture system A.R. Tracking and an immersive environment CAVE. The experiment consists of tracking the two arms of the patient with five markers: two for the left arm, two for the right arm and one for the head. Figure 4 represents a screenshot of our prototype showing the metaphor for virtual training and an application to manage the training progress. The control window is capable of opening a training motion in a database of Tai-Chi which is recorded and stored in a standard file called BVH (Bio-Vision Hierarchy). The control window provides a tool that allows choosing the viewpoint of virtual avatar (face-to-face or face-to-back) and the characteristics of avatar (point model, skeleton model, virtual human model, texture ...). The right corner presents the state of connection with motion capture system while the left corner shows the similarity between trainee's motion and the reference motion. When a training motion is completed, the similarity of both online mode and offline mode is returned for comparison. The background color is changed during the training according to the degree of similarity. This metaphor is very useful to guide the trainee in the execution of human gesture for motor rehabilitation. 


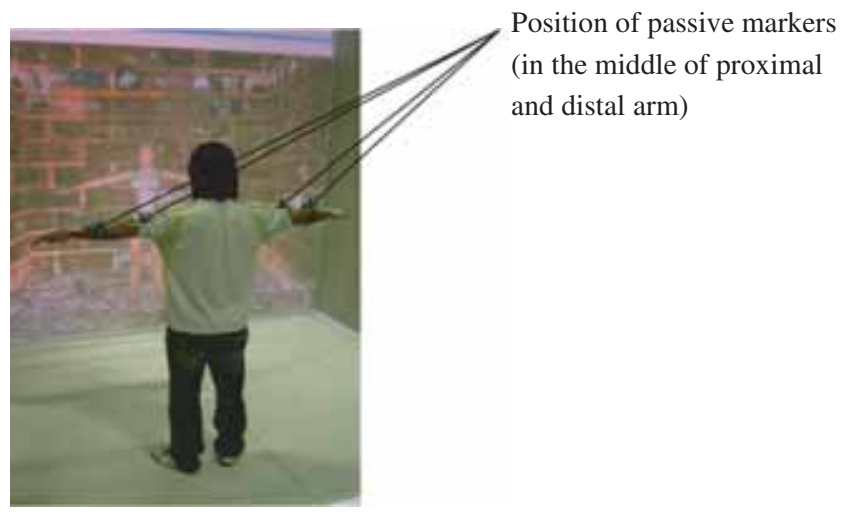

Fig. 4. Screenshot of training-based system for virtual rehabilitation in face to face mode.

The purpose of our experiments is to validate the rwLCSS algorithm. Therefore, we implemented three experiments. According to the work of Seongmin Baek et al. (2001), we fixed the experimental spatial thresholds to 10 degrees and 20 degrees for proximal-arm joints and distal-arm joints, respectively. ${ }^{2}$

\subsection{Motions tested}

To validate our algorithm, four different types of motion were used:

- Motion 1 (M1): non-mobile pose

- Motion 2 (M2): simple and slow motions

- Motion 3 (M3): slow Tai-Chi motions

- Motion 4 (M4): complicated and high velocity Tai-Chi motions

These motions are described as the following:

Motion 1 (M1): Non-mobile pose - This motion is not a movement due to the fact that the gesture is static. We can consider that a non-mobile pose is a motion with an infinite delay between gestures. This would be helpful for improving our algorithm and verifying its stability. Three experimented poses (noted M1a, M1b and M1c) was tested for the motion M1. They are illustrated in the Fig. 5.

Motion 2 (M2): Simple and slow motions - A simple motion means that the virtual avatar poses a simple position and moves very slowly so the trainee can follow the motion easily. Figure 6 shows key-frames series for the three different M2 motions (noted M2a, M2b and M2c).

Motion 3 (M3): Slow Tai-Chi motions - Tai-Chi motions are complex compared to motion M2. We first use slow and simple three Tai-Chi motions to enable the trainee to follow and replicate easily. Figure 7 shows some key-frames series of those motions (noted M3a and M3b). 

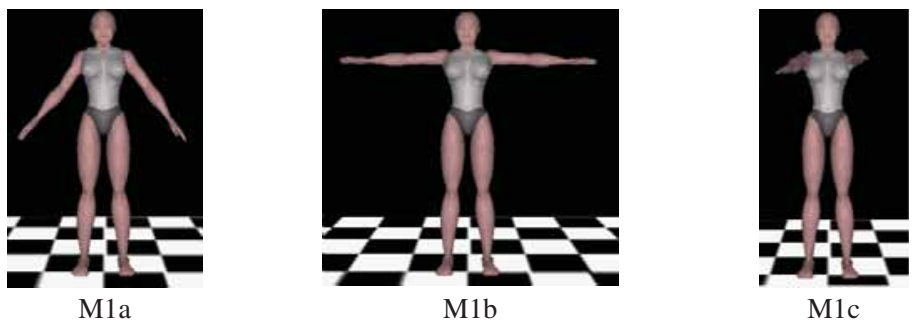

Fig. 5. Motion M1: Non-mobile poses (three different gestures M1a, M1b and M1c).

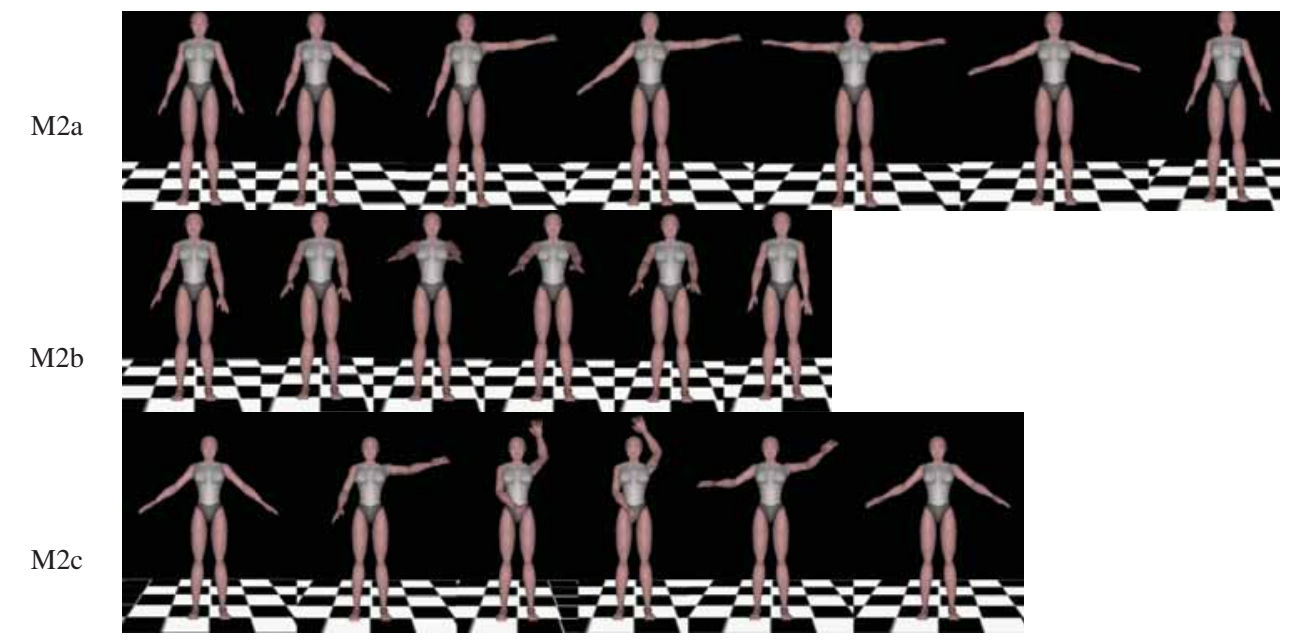

Fig. 6. Motion M2: Simple and slow motions (key frames of motions M2a, M2b and M2c).

M3a

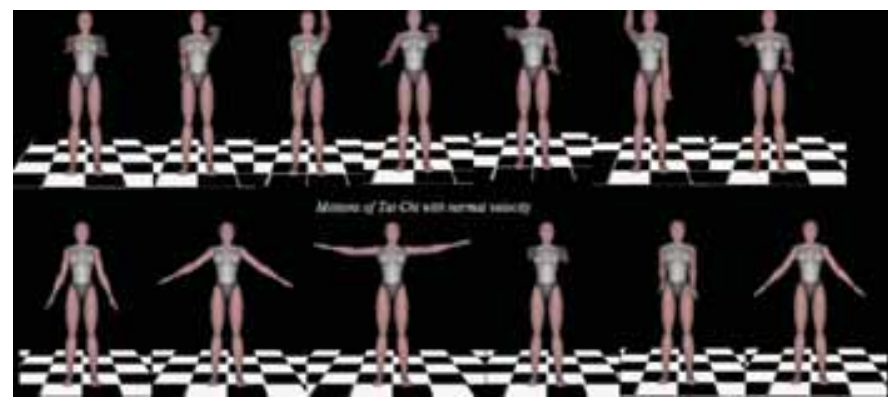

Fig. 7. Motion 3: Slow Tai-Chi motions M3a and M3b. 


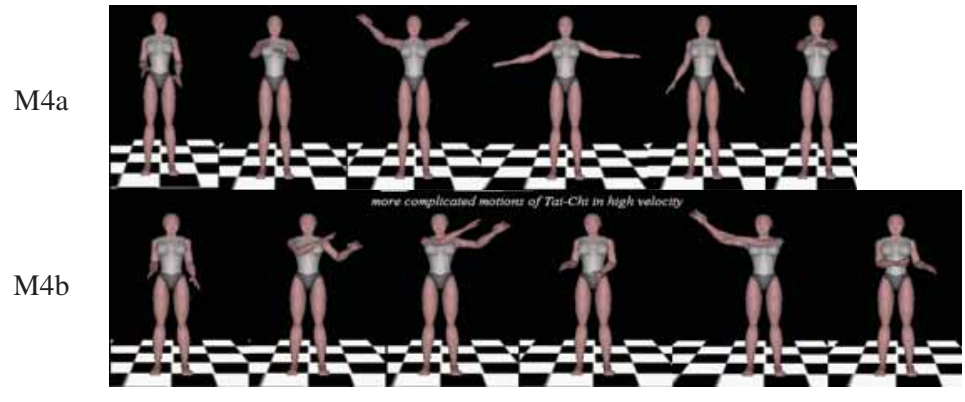

Fig. 8. Motion 4: fast Tai-Chi motions.

Motion 4 (M4): High velocity Tai-Chi motions - To confirm the validity and reliability of our algorithm, we use more complex and faster three Tai-Chi motions. Some key-frames of these motions are shown in Fig. 8.

\subsection{Experiments}

To validate the proposed rwLCSS approach, three different experiments were carried out. We tested the capability of our algorithm in terms of the similarity measurement between the motion made by a trainee and the motion executed by the avatar.

All experiments were carried out in a face to face mode which means the trainee faces the avatar as in front of a mirror and has to replicate a movement of the left arm of the avatar by a movement of his right arm (mirror metaphor).

The main objective of our experiment is to validate the stability of our proposed algorithm. Therefore we recruited nine healthy subjects aged 22-43 years (mean $30.4 \div 6.9$ ). All the participants are male and they belong to the staff of the Image Institute. All the participants are familiar with virtual reality and all tests were carried out using the MOVE system.

Experiment 1: No motion - In the first experiment, we tested the algorithm with the motions M1 (non-mobile gestures). The non-mobile gesture means that the virtual avatar poses the same position during the training. The purpose of this test is to evaluate the performance when the trainee poses the same position as the avatar.

Experiment 2: Reproducing simple motions - The second experiment consists in testing the algorithm with motion M2 (simple motions). The purpose of this test is to evaluate the performance of reproducing the motion when the trainee replicates very closely the motion of the avatar.

Experiment 3: Analysis the difference between the performances of doing simple motion and more complicated motion - The objective of the third experiment is to verify the difference between the performance of doing the simple 
motions and complicated motions. For this objective, we use two groups of motions M3 (simple motions) and M4 (more complicated motions).

\subsection{Results and discussion}

The validation of our rwLCSS algorithm is performed on the full body avatar model for all frames of the motion. We record the final performance of reproducing the motion. For each experiment, we present and discuss the obtained results.

Experiment 1: No motion - The result showed that the performances are at $100 \%$ for all non-mobile gestures for all subjects.

Experiment 2: Reproducing simple motions - In the second experiment, the result is shown in Fig. 9 is quite close to $100 \%$ for nine subjects trained with three simple and slow motions. Three curves in Fig. 9 illustrate the performances of doing three simple motions for nine subjects. The performance averages of doing this task for nine subjects doing three simple motions are $96,9 \div 1,7 \% ; 98,8 \div 1,0 \%$; $96,9 \div 1,0 \%$, respectively.

Experiment 3: The analysis the difference between the performances of doing simple motion and more complicated motion - Figure 10 shows the curves of obtained performances for six motions which are taken from two groups of motions M3 and M4.

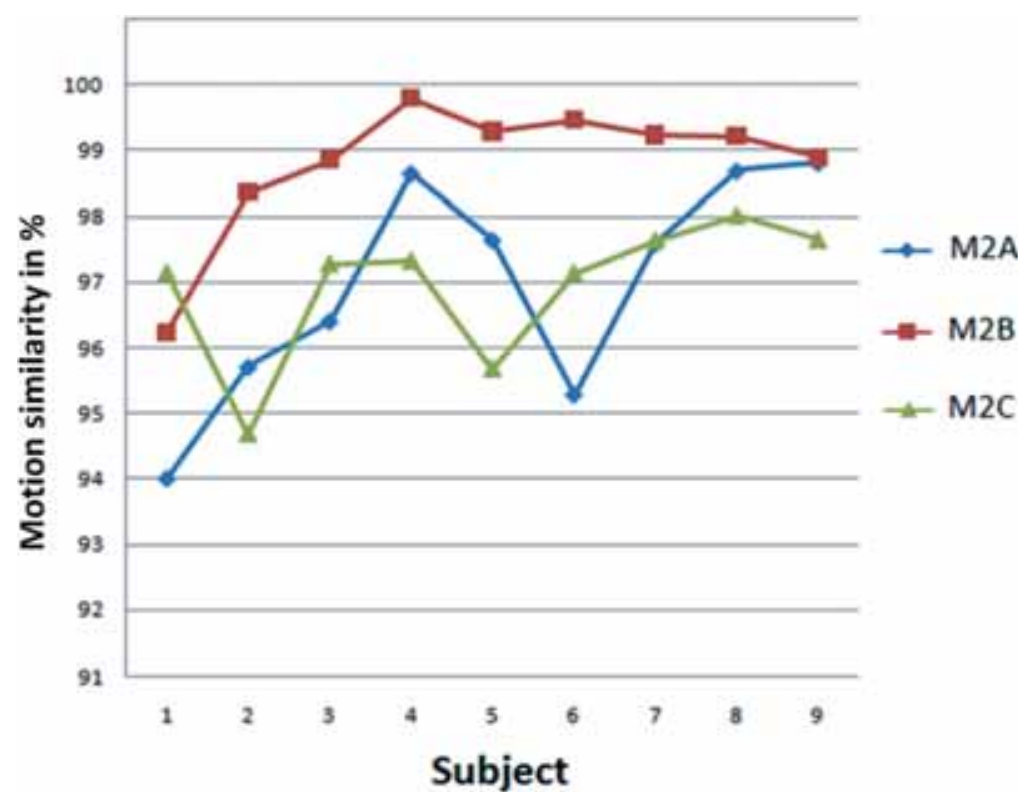

Fig. 9. Result for the second experiment: quite close to $100 \%$ of performance of reproducing the simple and slow motions. 


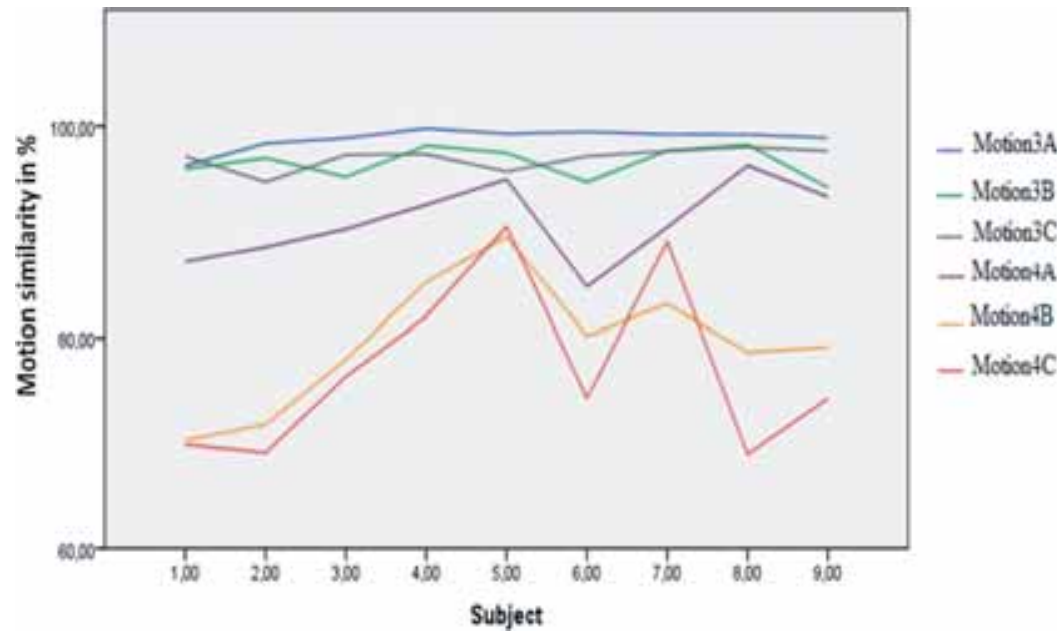

Fig. 10. Result for the third experiment: significant different between performances of doing simple motions and complicated motions.

Table 1. Using one-way ANOVA for the performance of reproducing motions.

\begin{tabular}{|c|c|c|c|c|c|c|}
\hline \multicolumn{7}{|c|}{ Multiple comparisons } \\
\hline \multicolumn{7}{|c|}{ Performance Tukey HSD } \\
\hline \multirow[b]{2}{*}{ (I) motion } & \multirow[b]{2}{*}{$(\mathrm{J})$ motion } & \multirow[b]{2}{*}{$\begin{array}{c}\text { Mean } \\
\text { difference (I-J) }\end{array}$} & \multirow[b]{2}{*}{$\begin{array}{l}\text { Std. } \\
\text { error }\end{array}$} & \multirow[b]{2}{*}{ Sig. } & \multicolumn{2}{|c|}{$95 \%$ confidence interva } \\
\hline & & & & & $\begin{array}{l}\text { Lower } \\
\text { bound }\end{array}$ & $\begin{array}{l}\text { Upper } \\
\text { bound }\end{array}$ \\
\hline \multirow{5}{*}{1.00} & 2.00 & 2.32222 & 2.07259 & 0.871 & -3.8290 & 8.4735 \\
\hline & 3.00 & 1.86667 & 2.07259 & 0.944 & -4.2846 & 8.0179 \\
\hline & 4.00 & $8.98500^{*}$ & 2.07259 & 0.001 & 2.8338 & 15.1362 \\
\hline & 5.00 & $19.28444^{*}$ & 2.07259 & 0.000 & 13.1332 & 25.4357 \\
\hline & 6.00 & $21.67000^{*}$ & 2.07259 & 0.000 & 15.5188 & 27.8212 \\
\hline \multirow{5}{*}{2.00} & 1.00 & -2.32222 & 2.07259 & 0.871 & -8.4735 & 3.8290 \\
\hline & 3.00 & -0.45556 & 2.07259 & 1.000 & -6.6068 & 5.6957 \\
\hline & 4.00 & $6.66278^{*}$ & 2.07259 & 0.027 & 0.5115 & 12.8140 \\
\hline & 5.00 & $16.96222^{*}$ & 2.07259 & 0.000 & 10.8110 & 23.1135 \\
\hline & 6.00 & $19.34778^{*}$ & 2.07259 & 0.000 & 13.1965 & 25.4990 \\
\hline \multirow{5}{*}{3.00} & 1.00 & -1.86667 & 2.07259 & 0.944 & -8.0179 & 4.2846 \\
\hline & 2.00 & 0.45556 & 2.07259 & 1.000 & -5.6957 & 6.6068 \\
\hline & 4.00 & 7.11833* & 2.07259 & 0.015 & 0.9671 & 13.2696 \\
\hline & 5.00 & $17.41778^{*}$ & 2.07259 & 0.000 & 11.2665 & 23.5690 \\
\hline & 6.00 & $19.80333^{*}$ & 2.07259 & 0.000 & 13.6521 & 25.9546 \\
\hline
\end{tabular}

*The mean difference is significant at the 0.05 level.

From the significant values of this table, the difference of performance between the simple motions group and complicated motions is very significant. All the significant values between simple motions and complicated motions group are far less than $p=0.05$. 
From these curves, we could notice that, the performance difference between the two groups is considerable. The subjects executed simple motions with better performance than the complicated motions. For more detailed analysis of the difference, we performed a one-way analysis of variance (ANOVA) on those performance curves by using the Tukey Honest Significant Difference (Tukey HSD) method. The result is described in Table 1 .

\section{Conclusion}

In this research, we proposed a simple but robust algorithm to measure in realtime the performance of trainees in replicating a motion represented by a virtual avatar in a virtual environment. The patient performance is measured by using key-frames approach and by processing simultaneously the trainee's motion and the reference motion. The first results obtained are promising for comparing two motions executed in the context of motor rehabilitation purpose.

The method will be applied for guiding patients to do practice functional rehabilitation exercises from simple to complicated motions. Therefore, we expect that our approach will be very useful in therapy domain for physical rehabilitation based on training task. The prototype system developed in this research is based on TaiChi motions which are very interesting to the field of rehabilitation because of their healthy.

\section{Acknowledgments}

This work is part of the project SIMACTION supported by the French Agency for the Research (ANR) through the call "Technologies for Health".

\section{References}

1. D Thalmann, "The role of virtual humans in virtual environment technology and interfaces," Proceeding Joint EC-NSF Advanced Research Workshop (1999).

2. S. Baek, S. Lee and G. Kim, "Motion retargeting and evaluation for vr-based training of free motions," The Visual Computer 19(4), 222-242 (July 2003).

3. V. Wank, A. Fischer, K. Bos, I. Boesnach, J. Moldenhauer and T. Beth, "Similarities and varieties in human motion trajectories of predefined grasping and disposing movements," Proceeding 2004 4th IEEE/RAS International Conference on Humanoid Robots 1, 311-321.

4. T. Yabe and K. Tanaka, "Similarity retrieval of human motion as multi-stream time series data," Proceeding 1999 International Symposium on Database Applications in Non-Traditional Environments.

5. M. Cardle, Automated Motion Editing, Technical report 2004, Computer Laboratory, University of Cambrigde.

6. H. Ding, G. Trajcevski, P. Scheuermann, X. Wang and E. Keogh, "Querying and mining of time series data: Experimental comparison of representations and distance measures," Proc. of the 34th VLDB, pp. 1542-1552.

7. M. Vlachos, G. Kollios and D. Gunopulos, "Discovering similar multidimensional trajectories," Proceeding of ICDE Conference (2002). 
8. H. Andre-Jonsson and D. Z. Badal, "Using signature files for querying time-series data," Proceeding of PKDD Conference (1997).

9. M. Vlachos, M. Hadjieleftheriou, D. Gunopulos and E. J. Keogh, "Indexing multidimensional time-series," Proceeding of VLDB 15(1), (2006).

10. E. J. Keogh, "Exact indexing of dynamic time warping," Proceeding of VLDB Conference (2002).

11. E. J. Keogh and C. A. Ratanamahatana, "Exact indexing of dynamic time warping," Knowl. Inf. Syst. 7(3), (2005)

12. E. J. Keogh and S. Kasetty, "On the need for time series data mining benchmarks: A survey and empirical demonstration," Data Min. Knowl. Discov. 7(4), (2003).

13. J. Cameron and J. Lasenby, "A real-time sequential algorithm for human joint localization," ACM SIGGRAPH Posters, 107 (2005).

14. A. Aristidou, J. Cameron and J. Lasenby, "Real-time estimation of missing markers in human motion capture," ICBBE 2008. The 2nd International Conference on Bioinformatics and Biomedical Engineering, 16-18 May 2008, 1343-1346.

15. A. Croitoru, P. Agouris and A. Stefanidis, "3D trajectory matching by pose normalization," Geographic Information Systems Proceedings of the 13th Annual ACM International Workshop on Geographic information Systems, Bremen, Germany.

16. http://www.videomassage.fr/les-18-positions-de-tai-chi-massage/

17. M. Vlachos, "Indexing time serie," Lecture in ICDM Conference (2006).

18. M. Oshita, "Motion-capture-based avatar control framework in third-person view virtual environments," ACM SIGCHI International Conference on Advances in Computer Entertainment Technology 2006 (ACE 2006), Hollywood, USA (June 2006).

19. P. Salamin, F. Vexo and D. Thalmann, The benefits of third-person perspective in virtual and augmented reality? Proceeding of the ACM Symposium on Virtual Reality Software and Technology (VRST '06), pp. 27-30 (2006).

20. M. Holden, E. Todorov, J. Callahan and E. Bizzi, "Virtual environment training improves motor performance in two patients with stroke: Case report," Neurology Report 23, 57-67 (1999)

21. L. Piron, M. Dam, E. Trivello, V. Iaia and P. Tonin, "Virtual environment training ameliorates motor deficits in post-stroke patients," Neurology 52, A138 (1999).

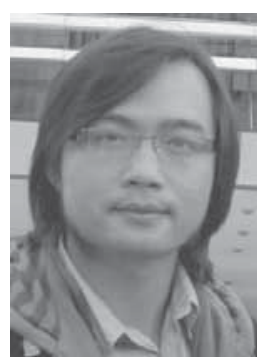

Van-Hanh Nguyen received his diploma in information technology engineering from Hanoi University of Technology (HUT), Hanoi, Vietnam in 2006. In 2007, he obtained his Master degree in Imaging, Vision and Robotic from Grenoble Institute of Technology, Grenoble, France.

$\mathrm{He}$ is currently working toward his $\mathrm{PhD}$ degree in Virtual reality, at Arts \& Métiers - ParisTech, Le2i, CNRS, ENSAM Imaging Institute — 71100 Chalôn Sur Saone, France. His current research interests include virtual rehabilitation, time-series data mining, machine learning, Bayesian-based methods and statistical signal processing. 

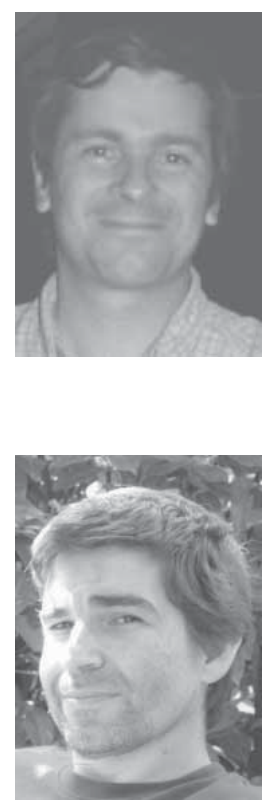

Frederic Merienne obtained his engineering degree from the Ecole Nationale Supérieure d'Arts \& Metiers in 1992. He obtained his $\mathrm{PhD}$ in January 1996 in electronics at the Institut National Polytechnique de Grenoble. Since 1996, he is a teacherresearcher at the Le2i, Laboratory of Electronic, Computer and Imaging Sciences, France. His research interests are focused on virtual immersion. He is currently professor at Arts \& Metiers ParisTech and director of the Imaging Institute.

Jean-Luc Martinez obtained his master degree in computer science from the University of Burgundy, Dijon, France in 1988 and received postgraduate degrees in computer graphics and artificial intelligence from the Ecole Nationale Supérieure des Mines de Saint-Etienne, France in 1989 and the Ecole Nationale Supérieure des Télécommunications de Brest, France in 1990.

From 1990 to 2005, Jean-Luc has worked in the field of $3 \mathrm{D}$ computer animation software industry for companies such as Thomson Digital Image, Softimage (Microsoft Corp.), Alias|Wavefront (Silicon Graphics Inc.) and Realviz.

Presently, Jean-Luc is a research engineer at the Imaging Institute (Arts \& Métiers - ParisTech, Chalon-sur-Saône, France). His current research interests include massive data visualization and parallel computing. 